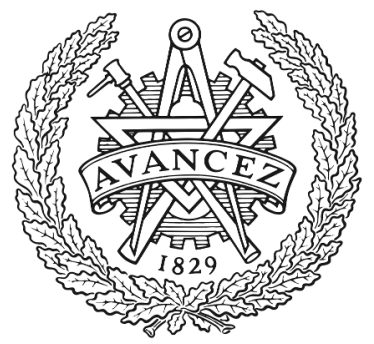

CHALMERS

UNIVERSITY OF TECHNOLOGY

\title{
Effect of Airfoil Parametrization on the Optimization of Counter Rotating Open Rotors
}

Downloaded from: https://research.chalmers.se, 2023-04-26 11:53 UTC

Citation for the original published paper (version of record):

Montero Villar, G., Lindblad, D., Andersson, N. (2019). Effect of Airfoil Parametrization on the Optimization of Counter Rotating Open Rotors. AIAA Scitech 2019 Forum.

http://dx.doi.org/10.2514/6.2019-0698

N.B. When citing this work, cite the original published paper. 


\title{
Effect of Airfoil Parametrization on the Optimization of Counter Rotating Open Rotors
}

\author{
Gonzalo Montero Villar*, Daniel Lindblad ${ }^{\dagger}$ and Niklas Andersson* \\ Chalmers University of Technology, Gothenburg, SE-412 96
}

\begin{abstract}
The present study compares two optimizations performed on Counter Rotating Open Rotors (CRORs) running at the same operating condition. The main difference between the two optimizations is the airfoil profile used to construct the blades. The first, uses the NACA 16 family of airfoils, whereas the second one, uses a parametrized airfoil type, CST. Two independent multi-objective optimizations are carried out using approximately the same computational resources. All the design variables except those concerning the airfoil profile, are kept with the same design freedom so that a fair comparison can be made. Both sets of configurations are aerodynamically optimized for maximum thrust coefficient and efficiency at top of climb conditions. The optimization is performed using multi-objective Differential Evolution (DE) coupled with 3D RANS simulations and Radial Basis Function (RBF) meta-modeling.
\end{abstract}

\section{Nomenclature}

$\begin{array}{ll}E A & =\text { evolutionary algorithm } \\ D E & =\text { differential evolution } \\ G A & =\text { genetic algorithm } \\ R B F & =\text { radial basis function } \\ C R O R & =\text { counter rotating open rotor } \\ L H S & =\text { latin hypercube sampling } \\ T & =\text { total thrust, } \mathrm{N} \\ P & =\text { total power, } \mathrm{W} \\ \eta & =\text { propeller efficiency } \\ C_{T} & =\text { thrust coefficient } \\ n & =\text { blade rotational speed, rev/s }\end{array}$

\section{Introduction}

$\mathrm{T}$ HE Counter Rotating Open Rotor (CROR) configuration has been widely studied over the past decades, as a promising technology to increase efficiency in comparison to current turbofan engines due to its ultra high bypass ratio. Since the presentation in the mid 80s of the SR7L propeller by NASA [1], where NACA 65 and NACA 16 blade profiles were used for the definition of the propeller geometry, these two airfoil families have been extensively used for propeller design. Stuermer et al. [2] studied the aeroacoustic and aerodynamic characteristics of a CROR configuration with blades composed of NACA 65, NACA 16, and a transition between both blade profiles. Capitao Patrao et al. used the NACA 16 family of airfoils for investigating the aerodynamic capabilities of a new type of propeller called the Boxprop [3, 4].

More recently, in order to optimize different type of turbomachinery components, efforts have been put into blades which do not use a given family of airfoils, but instead, use parametrized blade profiles in order to have more design freedom. For instance, Schnell et al. performed an optimization of a CROR configuration where both the acoustic and

\footnotetext{
*Ph.D. Student, Division of Fluid Dynamics, Department of Mechanics and Maritime Sciences, Chalmers University of Technology, SE-412 96 Gothenburg, Sweden

${ }^{\dagger}$ Ph.D. Student, Division of Fluid Dynamics, Department of Mechanics and Maritime Sciences, Chalmers University of Technology, SE-412 96 Gothenburg, Sweden

$\ddagger$ Assoc. Professor, Division of Fluid Dynamics, Department of Mechanics and Maritime Sciences, Chalmers University of Technology, SE-412 96 Gothenburg, Sweden
} 
aerodynamic performance were taken into account using parametrized blades [5] and Chen $e t$ al. used a different blade profile parametrization for a transonic compressor rotor optimization [6]. In 2006 Kulfan and Bussoletti proposed a generic parametrization capable of generating profiles for different aeronautical applications based on "class functions" (henceforth referred to as CST profiles) [7].

In the present work, two independent CROR multi-objective optimizations are carried out using Evolutionary Algorithms (EAs) together with 3D RANS simulations and Radial Basis Functions (RBFs). Both of them are aimed at maximizing propeller efficiency and thrust coefficient at top of climb conditions. For the design variables that are common to both optimizations, such as stacking line and chord distributions, the allowed design space is kept equal for both optimizations. The only difference is the airfoil shapes conforming the blades, one uses the NACA 16 family of airfoils, whereas the other one uses the method presented by Kulfan and Bussoletti [7]. These separate optimizations are done in order compare the performance obtained when using more complex parametrized blade profiles and the NACA 16 profiles when similar computational resources are available. Comparisons of the obtained pareto-fronts as well as analysis of the results are presented and discussed.

\section{Methodology}

The results in this work have been obtained using a Python based open-source optimization platform called HAMON. HAMON was originally developed in the Division of Fluid Dynamics at Chalmers University of Technology and is now freely available on-line via GitHub (https://github.com/gmonterovillar/HAMON][8]). HAMON's optimization methodology is based on evolutionary algorithms (either Genetic Algorithm (GA) or Differential Evolution (DE)) and can handle both single- and multi-objective as well as constrained and unconstrained problems. Use of meta-modeling is also available for problems where the evaluation of each design is too expensive, such as the 3D RANS simulations performed on the CROR configurations. For a more extensive description of HAMON and the methods it employs, the interested reader is referred to [9].

The optimization workflow consists of five main parts, namely; initial design set, geometry and mesh generation, CFD simulation, meta-model construction and optimization by means of EA. This process is illustrated in Fig. 1. Similar approaches where evolutionary algorithms are coupled with meta-models and CFD computations have been shown to work successfully for different types of turbomachines [4, 10, 12].

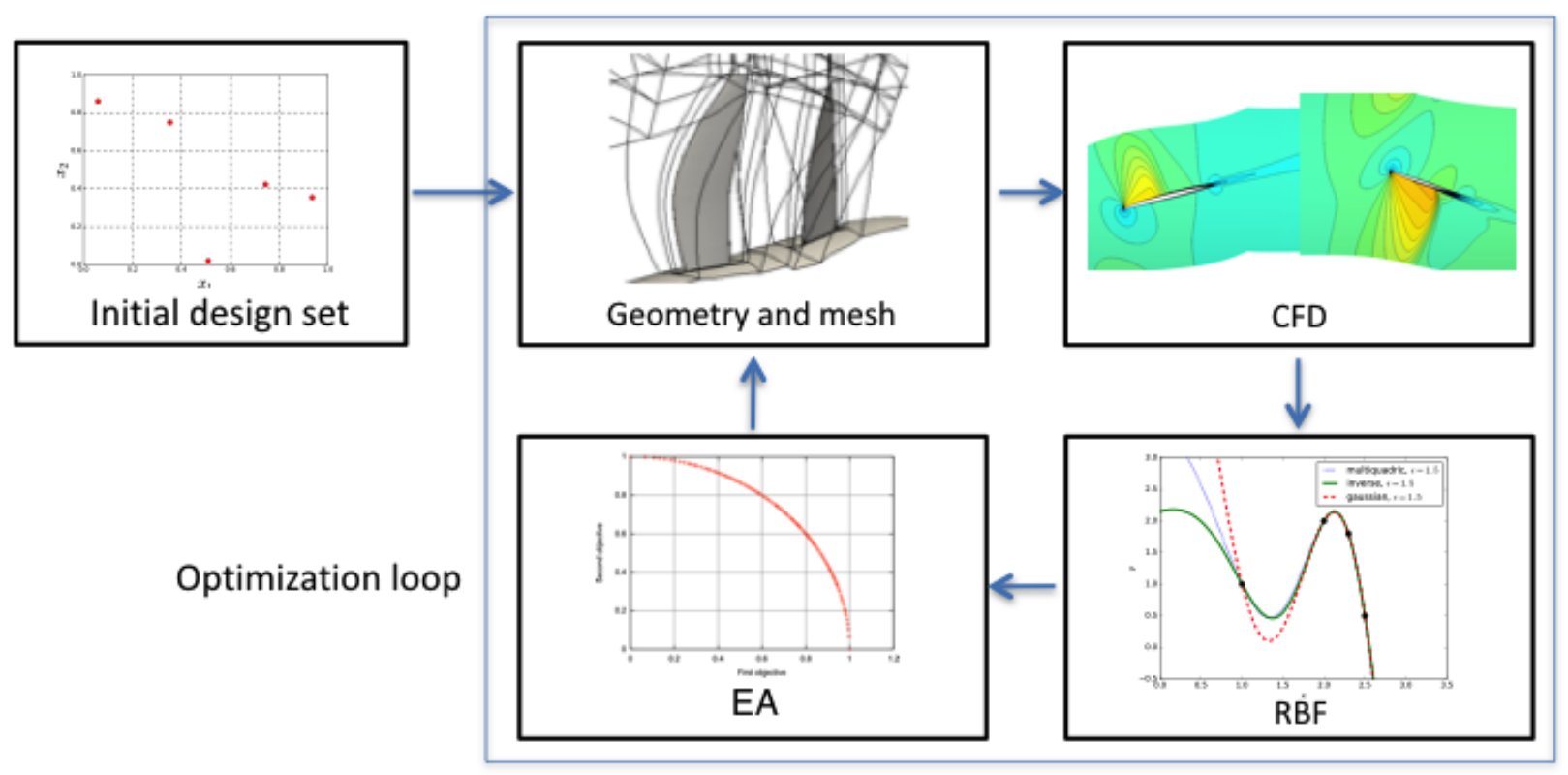

Fig. 1 Optimization procedure implemented in HAMON when RBFs are used.

In this five step process, first an initial sampling of the design space is done by means of Latin Hypercube Sampling (LHS) technique. Then, the main optimization loop is entered where the geometries and meshes for all the designs are generated and their objective functions are computed using 3D RANS simulations, namely; efficiency and thrust 
coefficient. With the obtained data, a response surface is constructed using a RBF. This response surface is passed to the EA as a base for the optimization. Finally the EA selects some designs along the found pareto-front (a set of optimal individuals in a multi-objective problem) and these are sent to the beginning of the optimization loop. These new individuals undergo the process of geometry and mesh generation as well as CFD evaluation. By doing this, more information is available for the RBF construction aiming at improving its prediction capabilities. This process is repeated until satisfactory results are obtained or the RBF is seen to converge to the CFD results.

\section{A. Geometry and mesh generation}

During both optimization processes, some parts of the CROR configuration are kept constant, these being; the hub contour, spacing between both rotors and the rear blade clipping. Some relevant design parameters which are common to both optimizations are presented in Table 1

Table 1 CROR design parameters.

\begin{tabular}{lc}
\hline Configuration & $12 \times 10$ blades \\
$D_{1}$ & $4.26 \mathrm{~m}$ \\
$D_{2}$ & $3.84 \mathrm{~m}$ \\
Hub to tip ratio (forward rotor) & 0.35 \\
Rotor-rotor spacing & $0.27 D_{1}$ \\
Airfoil & NACA $16 / C S T$ \\
\hline
\end{tabular}

In order for the EA to be able to optimize the CROR configurations, the entire geometry has to be parametrized using a finite number of design variables. In order to do so, radial distributions of different variables are defined at four different radial positions for each blade (hub, $60 \%$ of the span, $85 \%$ of the span and at the tip). With these four points given for every variable, Bèzier curves are constructed in order to obtain the entire radial distribution for each variable. These variables generate the chord distribution, the stacking line, distribution of angle of attack (with respect to the undisturbed flow) and the specific variables needed for either of the two blade profiles (see Sec. III.A.1 and Sec. III.A.2). Once all the distributions are generated, the blade geometry is computed by first obtaining the 2D blade profile, which is scaled with the chord and rotated according to the corresponding angle of attack. Finally the 2D profile is projected onto a cylindrical surface and placed so that the center of mass and the stacking line coincide. After all the airfoils have been stacked, the specified rotor geometry is obtained. This procedure is common to both optimizations and an example of this construction for one of the blades is illustrated in Fig. 2 a), where the stacking line together with the stacked airfoil sections can be seen.

From the CROR configuration geometry generation procedure just described, it can be seen that both optimizations have some common variables that define their geometry, these being chord, stacking line and angle of attack at the four defined positions. For these common variables the allowed range for both optimizations is the same so that a fair comparison can be made. The remainder of the parameters are the ones that define the $2 \mathrm{D}$ blade profiles and these are naturally different for the two blade types. The main differences between the two types are described below.

\section{NACA 16 blade profiles}

NACA 16 family of airfoils has been around for over half a century now and were initially developed for use in high-speed applications, especially propellers [13]. In order to obtain the geometric coordinates of one of these airfoils, two inputs are needed; a camber parameter $\left(c l_{i}\right)$ and a thickness parameter $(t)$. These two variables control the shape of the camber line and the thickness distribution respectively through the following relations (assuming the chord of the blade is unity, $0 \leq x \leq 1$ ),

$$
\begin{gathered}
y_{c}=-0.079577 c l_{i}[x \ln (x)+(1-x) \ln (1-x)] \\
t_{1}=0.01 t\left(0.989665 \sqrt{x}-0.23925 x-0.041 x^{2}-0.5594 x^{3}\right) \\
t_{2}=0.01 t\left(0.01+2.325(1-x)-3.42(1-x)^{2}+1.46(1-x)^{3}\right)
\end{gathered}
$$

where $y_{c}$ is the $y$ component of the camber line, and $t_{1}$ and $t_{2}$ are the thickness distributions when $x<0.5$ and $x \geq 0.5$ respectively. Once the camber and thickness distributions are obtained, the airfoil pressure and suction sides are drawn by putting them at a distance equal to the thickness at each point in the direction normal to the camber line. 
From the description above it can be seen that two parameters are required in order to obtain a 2D profile, $c l_{i}$ and $t$. Two more variables are needed in order to scale it to the appropriate size (chord) and rotate to the desired angle (angle of attack with respect to the undisturbed flow). This procedure leaves us with a total of four design variables per airfoil section when using NACA 16 profiles and a total of 44 for the entire CROR configuration. In this work, the trailing edge of the NACA 16 airfoils has been rounded.

\section{CST blade profiles}

The CST parametrization used for the construction of the CROR blades mostly follows the work by Kulfan and Bussoletti [7], where they presented a generic parametrization able to reproduce a wide range of airfoils, such as cambered airfoils and transonic airfoils. In this section a very brief discussion of the method is presented but for full details, the interested reader is referred to the work by the originals authors [7, 14]. Using this parametrization the following equations can be used to obtain the $y$ coordinate of the suction side $\left(y_{s}\right)$ of the airfoil at a given $0 \leq x \leq 1$ coordinate along the axial chord

$$
\begin{gathered}
S=\sum_{i=1}^{N} k_{i}\left(\begin{array}{c}
N \\
i
\end{array}\right) x^{i}(1-x)^{i} \\
y_{S}=S \sqrt{x}(1-x)+\left(\Delta z_{t e}+r_{t e}\right) x
\end{gathered}
$$

where $\mathbf{k}$ contains the scaling factors, $N$ is the order of the Bernstein polynomials, $\Delta z_{t e}$ is the offset of the trailing edge from the axial chord axis and $r_{t e}$ is the radius of the trailing edge. The first scaling factor can be directly related to another variable, the leading edge radius. Two more angles define the last scaling factor and control the orientation of the trailing edge. In a similar manner the pressure side can be obtained.

In order to try to alleviate the amount of variables needed for a 2D airfoil shape construction, some simplifications have been made in this work. Some of the parameters needed for the full representation are not considered to be optimization variables and are predefined at the four radial locations, namely; $\Delta z_{t e}, r_{t e}$ and both angles aforementioned. Some of these parameters that are kept constant control the camber of the profile, so in order to address this, the camber line definition from the NACA 16 (see Eq. 1) has been borrowed. The suction and pressure side coordinates obtained with Eq. 5 are interpreted as thicknesses in this work. Having a camber line and the corresponding thickness for the pressure and suction sides, a similar procedure to the one used for the NACA profiles is used in order to obtain the 2D airfoil. In total 6 scaling factors are used for both the pressure and suction side, but as previously mentioned, the first and last of them are dependent on other parameters (for instance, the first scaling factor is defined by the leading edge radius, which is considered to be a design variable). This approach results in 4 scaling factors needed for the suction side and another 4 for the pressure side as design variables, a camber parameter and a leading edge radius required in order to define the blade profile, plus chord and angle of attack in order to scale and rotate it. With this parametrization, a total of 108 design variables are required to define a CROR configuration.

\section{Computational mesh}

Since the design evaluation method used in this work is based on 3D RANS simulations, each design needs a computational mesh that represents its geometry. The generated geometry is used as an input for AutoGrid5 $5^{\mathrm{TM}}$ which is used for the mesh generation. Due to the large amount of simulations that are going to be performed, the cell count has been limited to approximately 1.7 million including both rotor blades and their corresponding domains. Once again, the mesh generation procedure is identical for both optimizations. An example of a generic CROR geometry construction by the described method and its corresponding mesh can be seen in Fig 2.

\section{B. Computational fluid dynamics}

In order to perform the 3D RANS simulations and solve the compressible Navier-Stokes equations, the commercial software STAR-CCM+ v12.04 is used. Steady state simulations at top of climb conditions are carried out where the turbulence is modeled using the one equation Spalart-Allmaras turbulence model [15] and the "all $y^{+}$wall treatment" option available in STAR-CCM+ is chosen to model the flow close to the walls. Several simulations were run before the actual optimizations began in order to determine the correct settings and it was found out that 5000 iterations were enough in order for the solver to be able to converge.

A sketch of the computational domain including specified boundary conditions can be seen in Fig. 3 . The domain extends 3.5 front rotor diameters from the centerline in height and 5.5 in the axial direction. The rotating domains are 

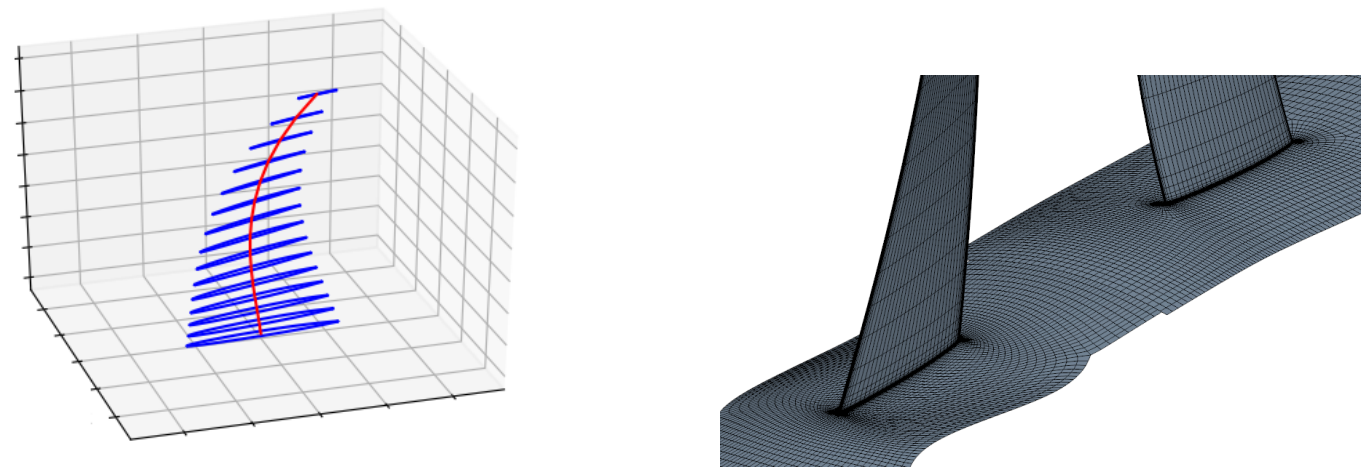

a) Stacking procedure (every other profile shown).

b) Computational mesh.

Fig. 2 Generic CROR configuration; geometry and mesh.

connected using a mixing plane and periodic boundary conditions are used at the pitch-wise boundaries allowing to include only one blade per row in the simulations.

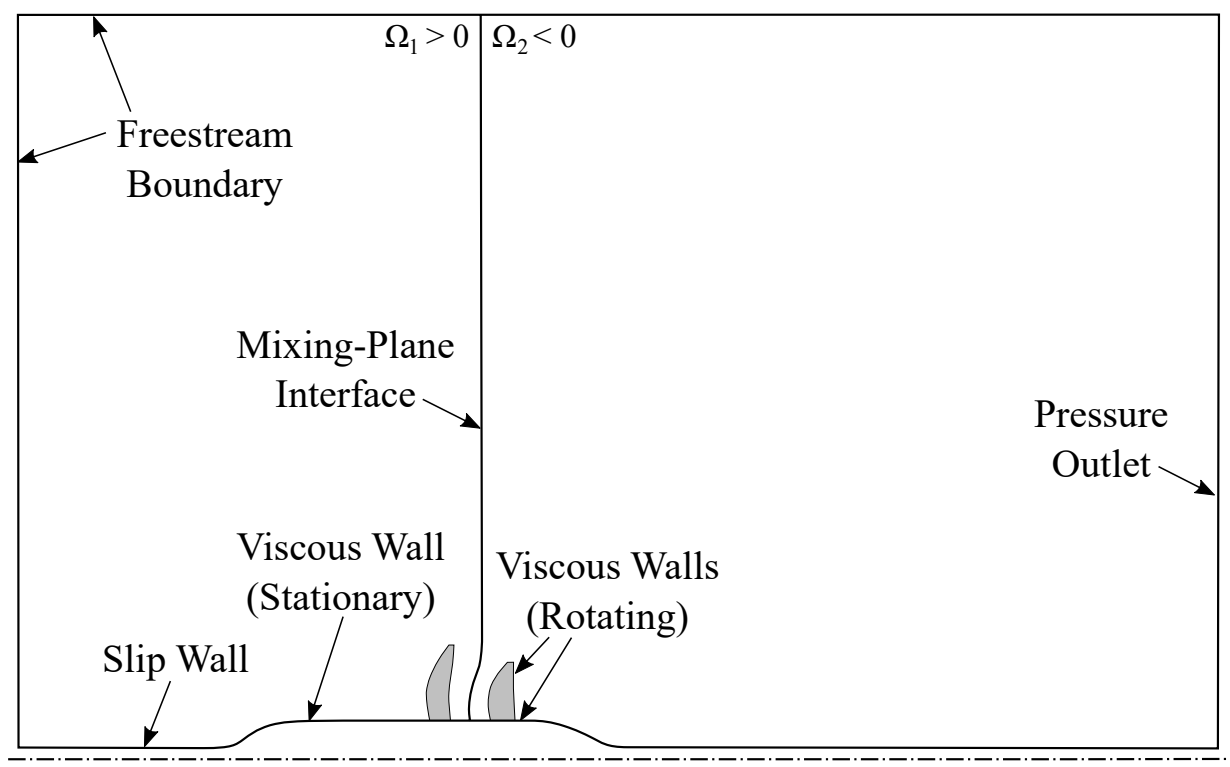

Fig. 3 A schematic overview of the computational domain including boundary conditions. Both blades and their corresponding hubs rotate.

The thrust coefficient $\left(C_{T}\right)$ and efficiency $(\eta)$ are used as objective functions for the optimizations. After each CFD simulation is completed the values of the these two objective functions are extracted and added to the database that is used by the RBF. These objective functions are computed as follows,

$$
\begin{gathered}
\eta=\frac{T V_{\infty}}{P} \\
C_{T}=\frac{T}{\rho_{\infty} n_{1}^{2} D_{1}^{4}}
\end{gathered}
$$

where $V_{\infty}$ is the free-stream velocity, $P$ the total power (both blades and corresponding rotating hubs are accounted for), $T$ is the thrust generated by the entire configuration accounting for nacelle drag, $\rho_{\infty}$ is the free-stream density and $n_{1}$ is the rotational speed of the front blade.

This optimization precess is done in an unsupervised manner, meaning that not all the hundreds of simulation are checked to verify their correct convergence. In order to address this issue and avoid contaminating the response surface 
with unrealistic values (values of simulations that have not converged properly), a convergence criteria has been imposed. After each CFD run, the root-mean-square deviation of the two objective functions (thrust coefficient and efficiency) over the last 1000 iterations is checked, if it is less than $0.1 \%$ the simulation is considered properly converged and the results are added to the data base of known designs, otherwise the results of that particular simulation are discarded.

\section{Meta-modeling}

The optimization method used by HAMON is based on evolutionary algorithms, which typically require more design evaluations than other more conventional approaches. A meta-model, in this particular case a radial basis function, fits a response surface to a set of known designs which allows predicting the performance of a new design without the need of a CFD simulation. This response surface can be evaluated in a computationally quick manner as it is an analytical expression where the resulting prediction depends on the Euclidean distance, in the design variable space, between the new configuration and the ones whose performance is already known. The analytical equation resulting from the RBF generation reads as follows,

$$
\begin{aligned}
\hat{y}(\mathbf{x}) & =\sum_{i=1}^{N} w_{i} \phi\left(r_{i}\right) \\
r_{i} & =\left\|\mathbf{x}-\mathbf{x}_{i}\right\|
\end{aligned}
$$

where $N$ is the number of designs for which the performance is known and used for the RBF construction, $\mathbf{x}_{\mathbf{i}}$ are the design variables for each of those designs, $\mathbf{x}$ are the design variables of the configuration for which the performance is to be predicted, $r_{i}$ is the Euclidean distance in the design space and $w_{i}$ are weights computed to ensure that the predicted performance for a known design matches the actual value. $\phi(r)$ is the basis function and several different ones are available, some common ones being

$$
\begin{aligned}
& \phi(r)=\sqrt{\left(\frac{r}{\epsilon}\right)^{2}+1} \\
& \phi(r)=\frac{1}{\sqrt{\left(\frac{r}{\epsilon}\right)^{2}+1}} \\
& \phi(r)=e^{-\left(\frac{r}{\epsilon}\right)^{2}}
\end{aligned}
$$

referred to as multiquadric, inverse and gaussian respectively and $\epsilon$ is a parameter that needs to be tuned. The basis function and $\epsilon$ parameter chosen can have a great impact on the shape of the response surface for a given data set. In Fig. 4 a simple example where the problem consist of only one design variable $x$, is used to illustrate how the choice of basis function for a given $\epsilon$ (Fig. 4 a)) or how the choice of $\epsilon$ for a given basis function (Fig. 4 b)) affects the response surface. The response surfaces shown in Fig. 4 are constructed using the same information, this being the $x$ and $y$ values for four known designs represented by black dots.

In order to find a suitable combination of $\epsilon$ and basis function to construct the response surface, the following procedure is followed in HAMON. First the entire database is split into two sets, one being used as the known designs in Eq. 8, and the second one used in order to be able to measure the prediction capabilities of each basis function/ $\epsilon$ combination. In this work the three different basis functions given in Eq. 9 are allowed, meaning that each basis function is used to construct a response surface (with the part of the data set that has been chosen to be used for construction) and the $\epsilon$ parameter is tuned. In order to be able to find a suitable $\epsilon$ for each of the basis functions, the second part of the data set is used, evaluating the response surface created with a given basis and $\epsilon$ on those designs, the error can be computed and thus the accuracy can be quantified. Now that an $\epsilon$ value can be associated with an error measure, this parameter can be tuned independently for each of the three basis functions. Once the three radial basis functions are constructed and tuned, they are compared with each other and the one with better accuracy is passed to the EA. An example of the resulting three RBFs after the tuning of $\epsilon$ can be seen in Fig. 5. being inverse the one yielding the best fit to the data.

Note that this response surface construction and tuning process is done once per objective function, so in this work one RBF is used for the prediction of the thrust coefficient and another one for efficiency. Every time new designs are evaluated in CFD inside the optimization loop depicted in Fig. 1 the tuning process is performed. 


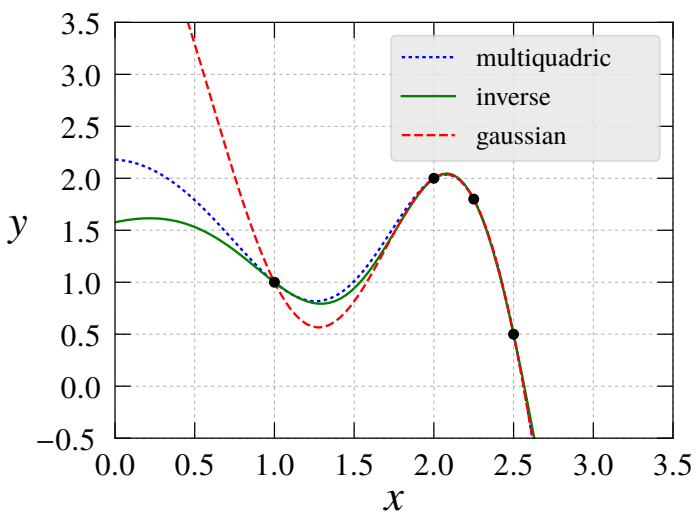

a) Effect of basis function (all $\epsilon=1.5$ ).

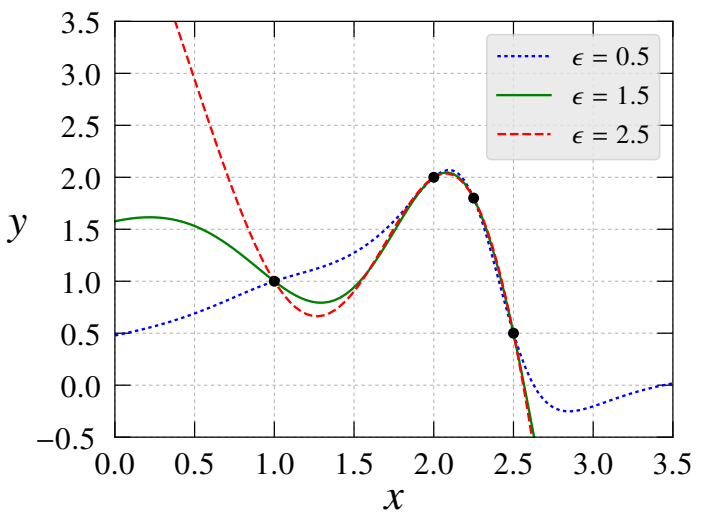

b) Effect of $\epsilon$ (all RBFs are of type inverse).

Fig. 4 RBF parameter choice, black dots represents the known points.

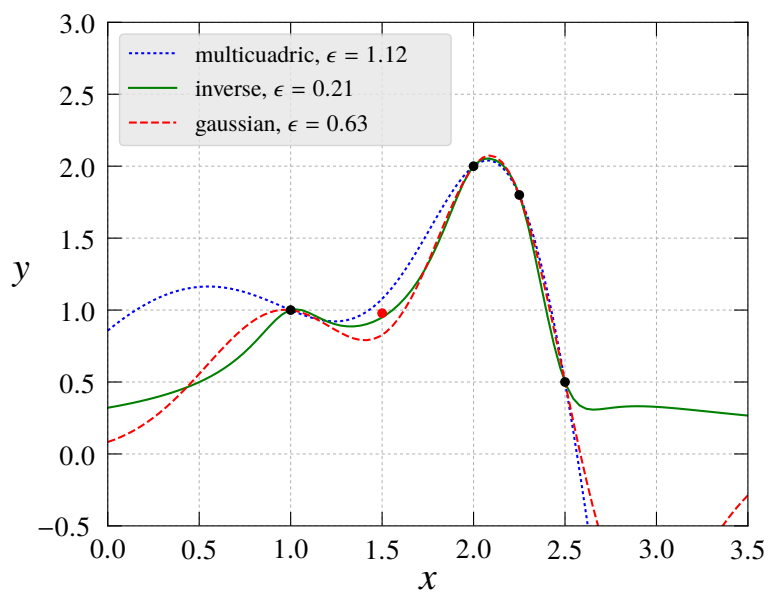

Fig. 5 Comparison of the three different basis functions with their corresponding tuned $\epsilon$ values. The black dots represent the data used for the RBFs construction and the red dot the data used for tuning.

\section{Evolutionary algorithms}

Evolutionary algorithms are stochastic optimization methods that try to mimic the evolution process observed in nature. Differential evolution belongs to this category and it is based on advancing a population of individuals from generation to generation through three main processes; mutation, recombination and selection. The first two of these processes are identical when dealing with single- and multi-objective optimization, but the third one is not. The main reason why multi-objective problems need different treatment for selection is that the comparison between two individuals is not as straight forward as it is in single-objective optimization. Another difference is that in single-objective problems the result of the optimization is one individual, whereas for multi-objective problems it is a set of individuals called pareto-front.

In order to perform the optimizations in this work, the multi-objective differential evolution available in HAMON is used. It uses standard differential evolution processes for mutation and recombination [16] and the selection/elitism is borrowed from the NSGA-II algorithm [17]. For a detailed explanation of how this is implemented in HAMON the interested reader is referred to [9].

To validate the method that is used for the CROR optimizations (illustrated in Fig. 1), where radial basis functions are used, the framework is tested on a well known function commonly used as a unconstrained multi-objective benchmark 
problem, the ZDT 1 function [18], which definition is given in Table 2 .

Table 2 Definition of ZDT 1.

\begin{tabular}{lccc}
\hline Function & variables, $\mathrm{m}$ & Variable range & Functions to minimize $\left(f_{1}(\mathbf{x}) \& f_{2}(\mathbf{x})\right)$ \\
\hline ZDT 1 & 30 & {$[0,1]$} & $f_{1}(\mathbf{x})=x_{1}$ \\
& & & $f_{2}(\mathbf{x})=g(\mathbf{x})\left[1-\sqrt{x_{1} / g(\mathbf{x})}\right]$ \\
& & $g(\mathbf{x})=1+9\left(\sum_{i=2}^{m} x_{i}\right) /(m-1)$ \\
\hline
\end{tabular}

The initial LHS contained 250 samples. After these have been evaluated, the first iteration of the RBF is generated (labeled "LHS" in Fig. 6, which is passed to the multi-objective DE to optimize on. The settings used for the optimization were; a population of size 70 and after each loop 25 individuals along the pareto-front were selected and evaluated again, in order to increase the amount of information available to the RBF for its construction. With the results of the evaluation of the new individuals added to the previous database (now containing a total of 275 evaluations) a new RBF is constructed and passed to the DE. This process is repeated a total of 6 times. The evolution of the prediction capabilities of the RBF can be seen in Fig. 6 , where it is clear that the more information is added to the RBF construction, the closer it gets to the true pareto-front, bringing the RBF closer to convergence. It is worth noting that the pareto-fronts shown in Fig. 6represent the fronts found by the DE using the RBF, not by evaluating the analytical expressions in Table 2. This explains why in Fig. 6b), some individuals can be seen to have lower objective function values than the ones on the true pareto-front.

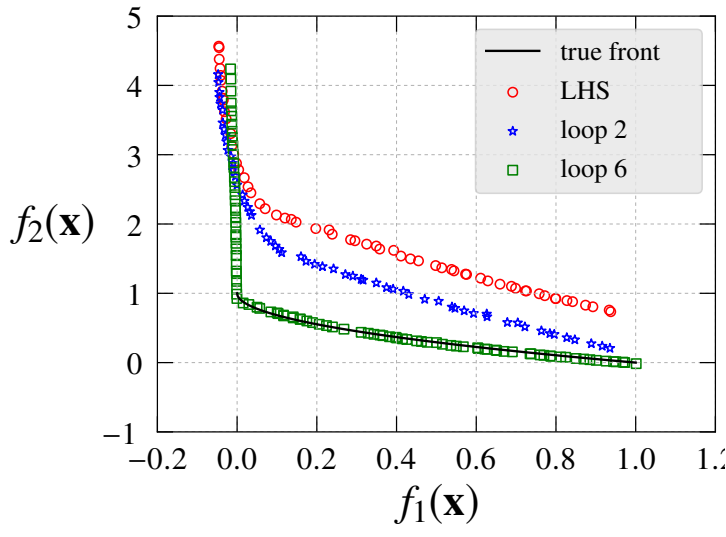

a) Entire pareto-front

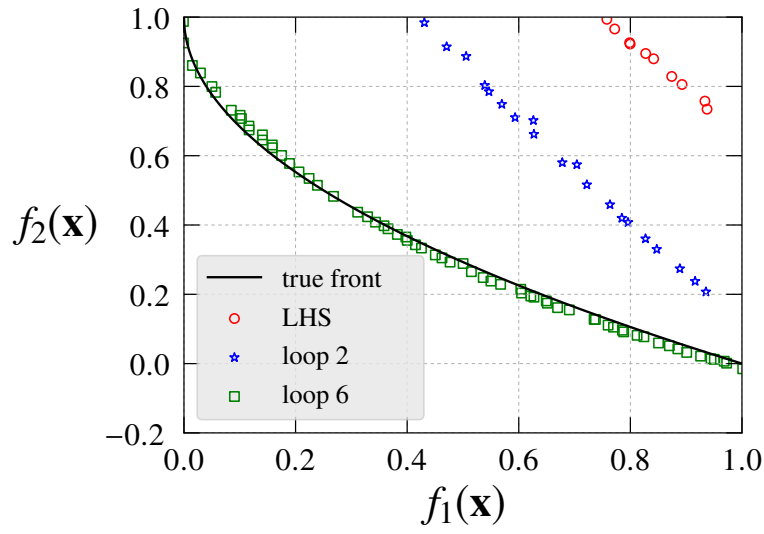

b) Close up view

Fig. 6 Evolution of the DE pareto-front over several optimization loops.

\section{Results}

The optimization framework which methodology is represented in Fig. 1 1 has been used to perform two independent CROR optimizations utilizing different blade profiles, NACA 16 and CST, at top of climb conditions, where thrust coefficient and efficiency were to be maximized. In order to parametrize the entire CROR configuration, 44 variables are needed when using the NACA 16 profiles and 108 when using CST. The hub, blade spacing and rear blade clipping are kept constant. Those variables that are needed in both optimizations (the chord, angle of attack and stacking line) are allowed to lay withing the same design range. It is important to note that both optimizations have been allowed to use similar amount of computational resources.

Initially a LHS with 900 samples is generated and a first RBF is built. Note that not all these 900 simulations have been considered when generating the response surface, as some of them did not fulfill the convergence criteria imposed due to the unsupervised nature of the method used. This was done to prevent introducing errors in the RBF by using data of simulations that were considered not to be properly converged. Multi-objective differential evolution was 
chosen as the optimization method, choosing a population size of 300 which run for 300 generations. In order to get the population size and number of generations, some initial tests were performed with the data obtained from the LHS to see what seemed to be the minimum requirements for obtaining a converged pareto-front from the optimizer. It was found that choosing 300 for both of those settings was sufficient and on the conservative side, which was not considered to be a problem, since the time and computational resources consumed by the optimizer are almost negligible when compared to those used when evaluating a design with CFD.

During the entire optimization processes, 55 optimization loops were performed, selecting 10 individuals from the pareto-front (obtained by the optimizer using the RBF) to be evaluated using CFD. These optimization loops together with the initial LHS resulted in a total of 1450 CFD evaluations for each of the optimizations. The optimization loops were divided into two groups, those aiming at exploring the design space, and those aiming at uniformly filling the pareto-front. This was achieved by changing the way in which individuals were selected from the predicted pareto-front in order to be evaluated using CFD. If exploration was sought for, 10 individuals were selected by comparing the crowdedness around them on the design space, whereas for filling the pareto-front the crowdedness was checked in the objective function space. These selection was done taking into account all the individuals from the last generation obtained with DE together with the already existing database of designs evaluated with CFD.

All these settings and parameters described above are common to both the NACA 16 and the CST optimizations, none of which had an initial baseline design. It is also important to note that same amount of CFD evaluation are allowed for both optimization in order to get an equal use of computational resources.

\section{A. Results from the NACA 16 optimization}

In order to evaluate the result of the optimization, the first thing to check are the locations of the designs in the objective function plane. In Fig. 7 all the evaluated designs that fulfill the convergence criteria can be seen, categorized in to three groups; the ones belonging to the LHS, the intermediate ones and the ones conforming the pareto-front when all the designs are included. It is clear from this figure that the optimization has allowed to improve the designs obtained from the LHS by increasing both efficiency and thrust coefficient.

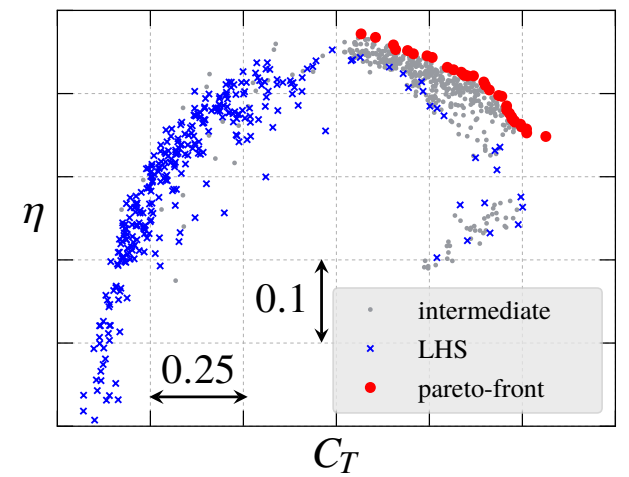

a) All evaluated configurations.

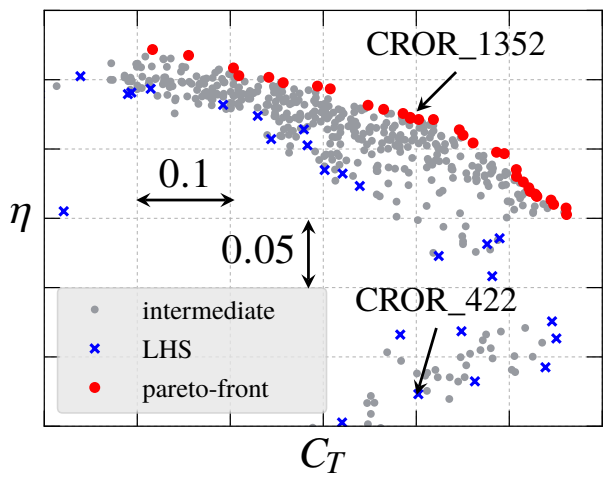

b) Close-up view of the pareto-front.

Fig. 7 Optimization result for the CROR with NACA 16 blade profiles.

As previously mentioned, this optimization has been carried out without a starting baseline design, hence the allowed ranges for the design variables were quite broad. In order to see if the chosen ranges are flexible enough and to see if it would be possible to narrow them down, or even fix some design variables for future optimizations, some trends are sought for. For instance, in Fig. 8 the extremes of the allowed design space for the stacking line, together with the stacking lines of all the pareto-front designs are shown. In Fig. 8 a) there is a clear trend on how the stacking line should look like according to the parametrization and optimization carried out in this work. This means that the variable ranges could have been chosen differently in order to make the optimization search easier, but at the same time shows that none of the pareto-front designs are hitting the limits, which means that the optimization has not been constrained by the chosen ranges. 


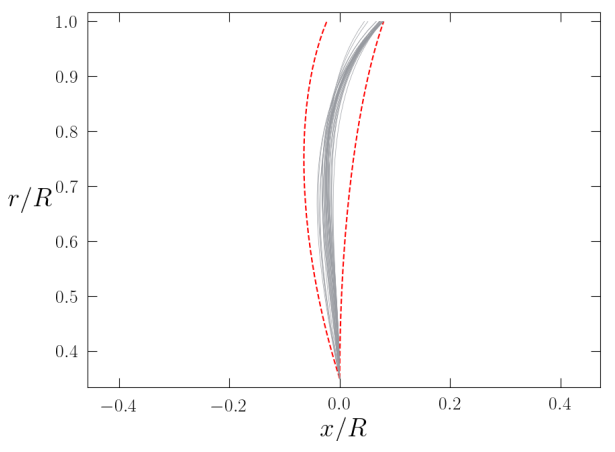

a) Meridional plane view, flow from left to right.

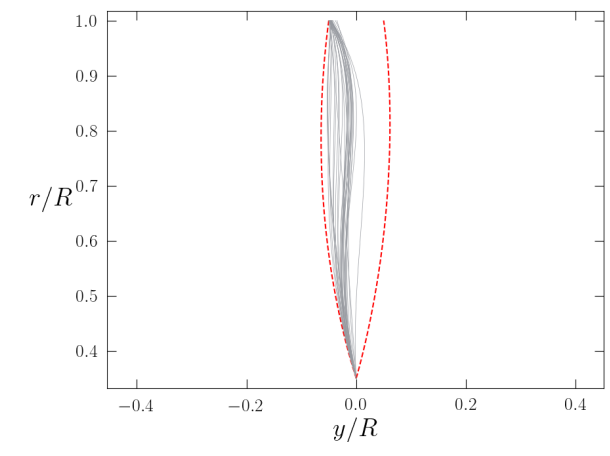

b) Azimuthal-radial plane view, blade rotating counterclockwise.

Fig. 8 Design space from the front rotor stacking line. - - encloses the allowed design space and - stacking lines from the designs belonging to the pareto-front. CRORs with NACA 16 profiles.

As can be seen in Fig. 7b), two designs have been selected, CROR_442 and CROR_1352. The first one belongs to the initial design set generated with the LHS and the second one is a design with the same thrust level that belongs to the pareto-front. It can be seen from this figure that the improvement in efficiency is quite noticeable. Lets take a closer look on these two designs in order to try to understand some of the changes that HAMON has been able to make to increase the efficiency of the CROR configuration.

Figure 9 shows contours of relative Mach number at $75 \%$ of the span for both selected configurations. It is quite evident that HAMON has been able to relocate the sock waves on CROR_1352 bringing them closer to the trailing edge. This new shock position not only favors the loading on the blades making the shocks weaker but also helps avoid what seems to be a shock induced separation visible in the suction side of the front rotor of the CROR_422 configuration.

\section{B. Results from the CST optimization}

Taking a look at Fig. 10, the improvements made by the optimizer when it comes to performance of the CST based CRORs can be seen represented in the objective functions plane. As previously mentioned for the NACA 16 optimization, all evaluated designs that fulfill the convergence criteria are plotted divided into three groups; LHS, intermediate and pareto-front.

Similarly as for the NACA 16, no baseline design was used for the optimization of the CROR. Taking this into account, the ranges for the design variables were given in a conservative manner to make sure that the optimization was not obstructed by the ranges chosen. Once again, some trends or patterns are sought for to see if a reduction of the variable ranges is possible and thus a speed up of the optimization. A very clear trend is again found for the stacking line, specially in the meridional plane projection, which can be seen in Fig. 11 Almost all the individuals from the pareto-front have a constant stacking line in the meridional plane, which suggests that maybe locking down the parameters responsible for that part of the design of the CROR configurations might have been a good approach, assuming this information was available before the optimization. It is seen again, that the conservative approach on the variable ranges worked, as none of the pareto-front design is hitting the limit of the design space they have available.

Two designs have been selected among all the evaluated ones which have been obtained at different stages of the optimization process, with very similar thrust coefficients, these being CROR_990 and CROR_1341 (see Fig. 10 b)). The thrust coefficients of the selected configurations are the same as for those obtained from the designs selected for the NACA 16 optimization (see Fig. 7b)). The relative Mach number contour at $75 \%$ of the span of the front rotor can be seen in Fig. 12, where it can be observed how the optimizer has been able to have a considerable impact in the shock waves. In this particular comparison the change that stands out the most is how the shock wave of the rear rotor blade has been drastically weakened. 


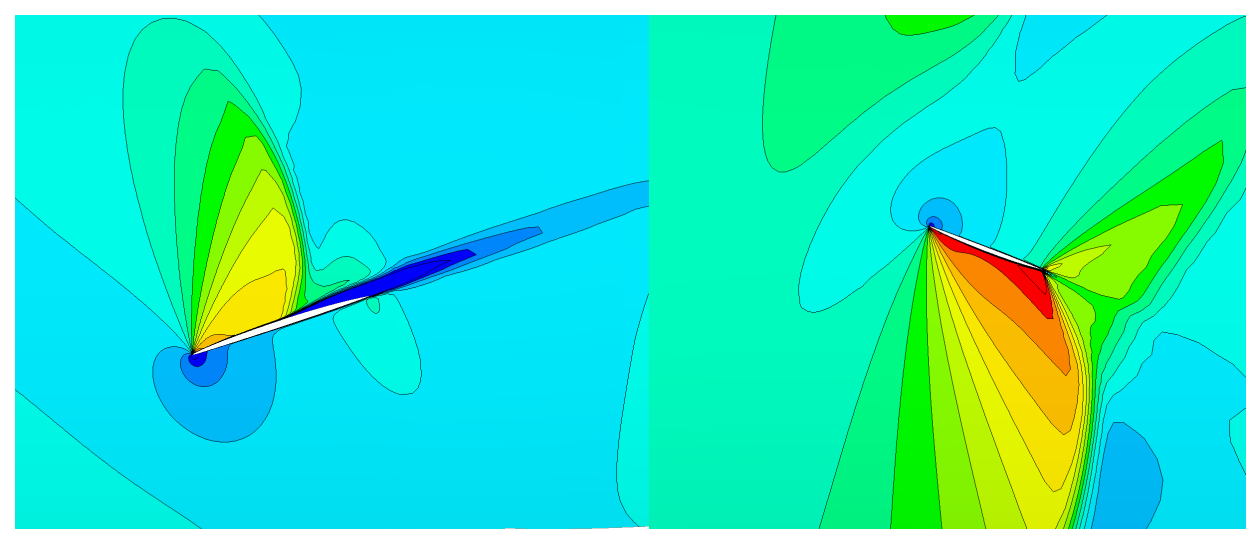

a) CROR_422

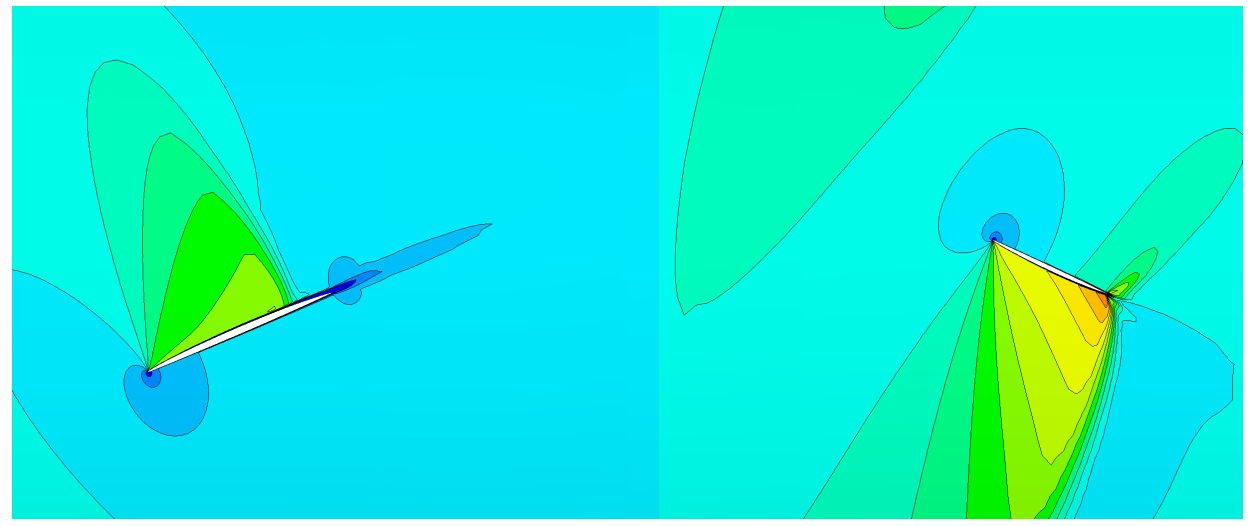

b) CROR_1352

Fig. 9 Relative Mach number at $75 \%$ span of the front rotor (same scale used). Flow from left to right. CRORs with NACA 16 profiles.

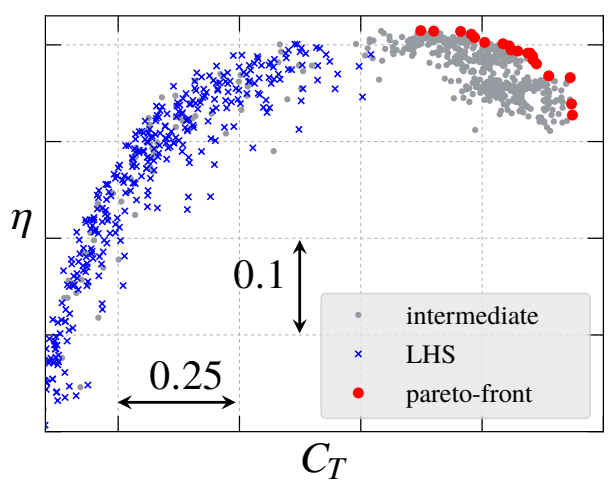

a) All evaluated configurations.

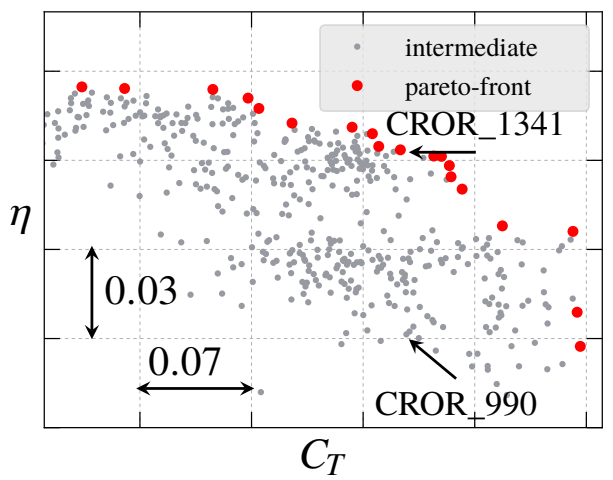

b) Close-up view of the pareto-front.

Fig. 10 Optimization result for the CROR with CST blade profiles.

\section{Comparison between both optimizations}

Now that both optimizations have been presented individually, some comparison between them can be made. The result of plotting the individuals belonging to the pareto-front of each of the optimizations side by side can be seen in 


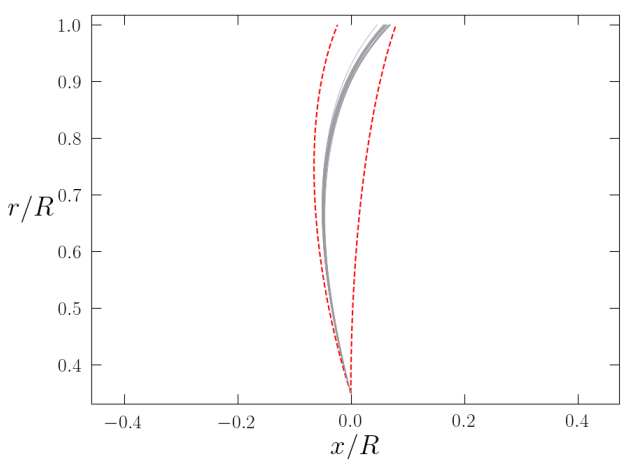

a) Meridional plane view, flow from left to right.

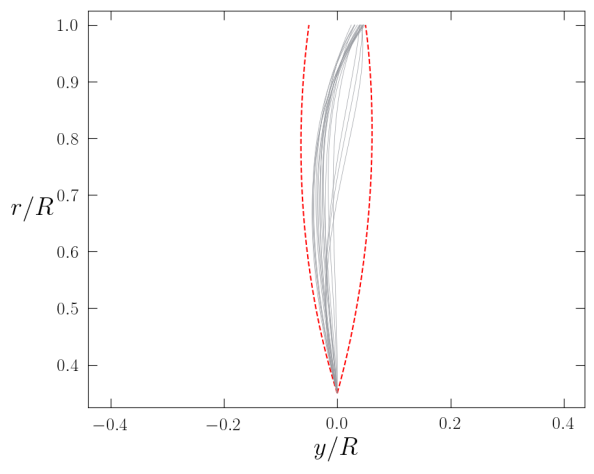

b) Azimuthal-radial plane view, blade rotating counterclockwise.

Fig. 11 Design space from the front rotor stacking line. - - encloses the allowed design space and - stacking lines from the designs belonging to the pareto-front. CRORs with CST profiles.

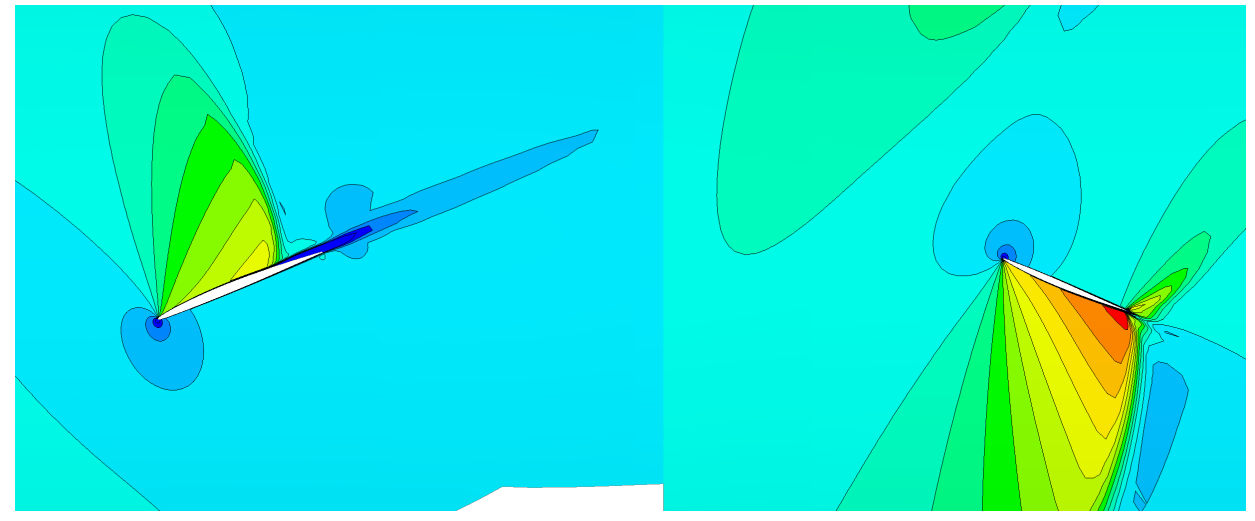

a) CROR_990

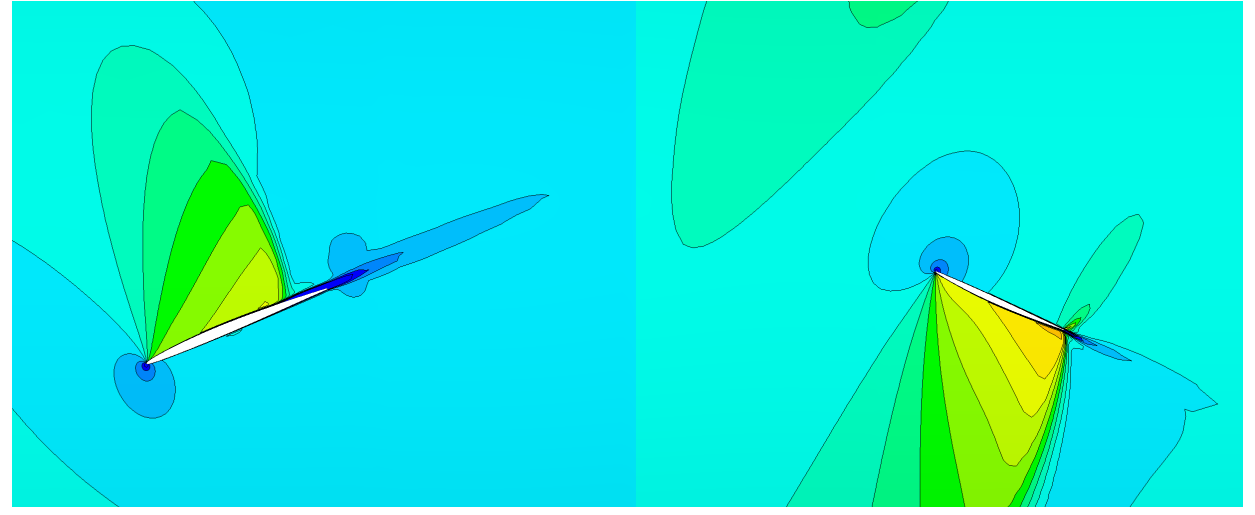

b) CROR_1341

Fig. 12 Relative Mach number at $75 \%$ span of the front rotor (same scale used). Flow from left to right. CRORs with CST profiles.

Fig. 13, where it is clear that the NACA 16 optimization has outperformed the CST one by approximate $2-3$ percentage points in efficiency on average. 


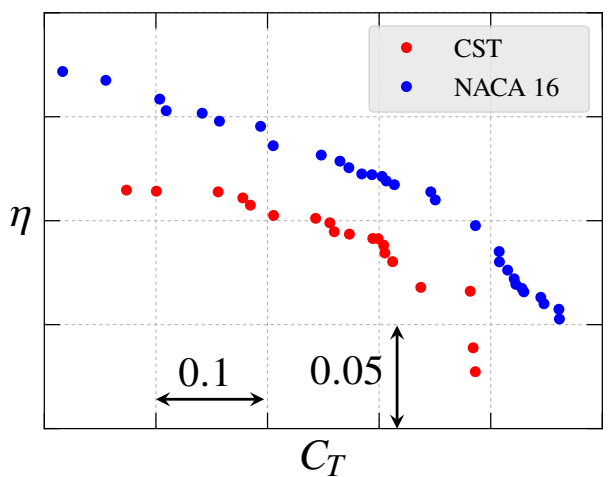

Fig. 13 Comparison of the obtained pareto-fronts for the two optimizations.

This might seem counter-intuitive, as one might tend to think that an airfoil shape with more design freedom, CST profiles, should be able to perform better or at least equally well as the more constrained one, NACA 16. Since both optimizations were given the same amount of computational resources, it seems reasonable to check how both optimization were doing at the time they were stopped, and an example is illustrated in Fig. 14. In this figure it can clearly be seen two very different behaviors on the prediction capabilities of the RBFs at the moment when the optimizations were stopped. Figure 14 a) shows that when the NACA 16 blade profiles were used, the response surfaces generated by the RBFs remained almost constant over the last five iteration loops, whereas Fig. 14b) tells us that when using CST profiles the RBF is still learning about the real shape of the response surfaces as a considerable change can be seen.

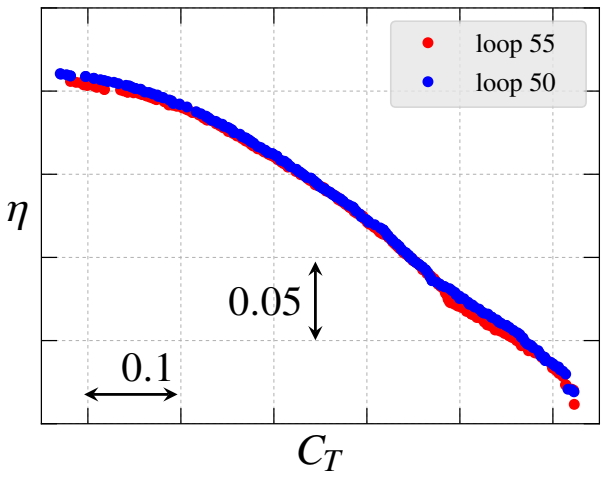

a) CROR with NACA 16 profiles.

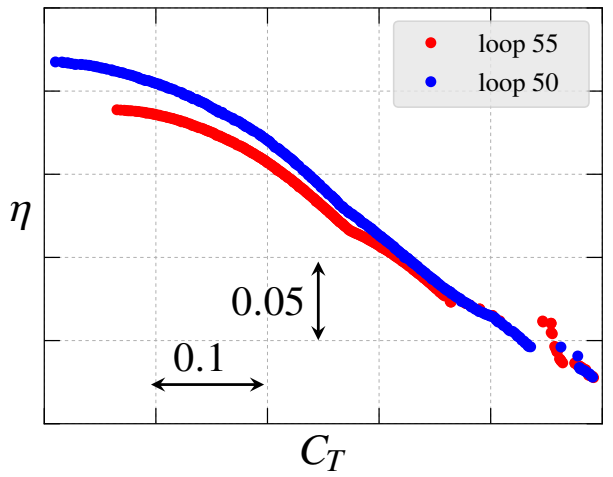

b) CROR with CST profiles.

Fig. 14 Pareto-fronts found by DE based on the RBF prediction at different stages of the optimization.

From the above discussion we can conclude that the CST optimization might have been stopped prematurely. Another interesting thing to look at is the evolution of the pareto-fronts obtained based on the CFD evaluations over the last optimization loops, instead of the one predicted using RBFs. The result of this comparison can be seen in Fig. [15]and it shows that the NACA 16 optimization has not been able to really improve the pareto-front but the CST optimization is continuously improving over the last five loops.

The reason for the different behaviors of both optimization can be associated with the dimensionality of both problems. Just to illustrate how the number of design parameters can affect the amount of required CFD evaluations, a simple calculation can be done. Equation 10 computes the minimum amount of designs needed in order to be able to obtain a second order polynomial in an $\mathrm{N}$-dimensional space, 


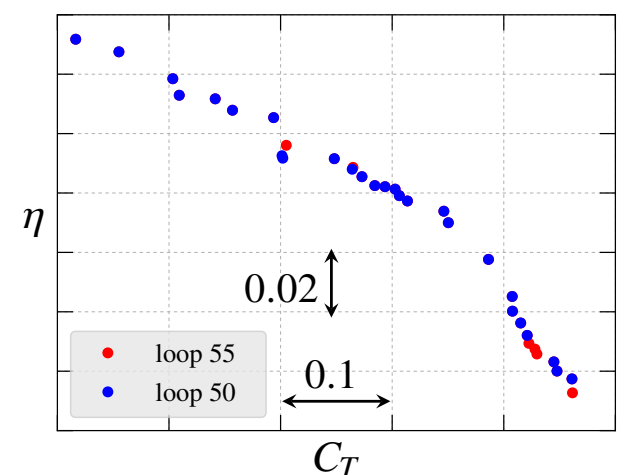

a) CROR with NACA 16 profiles.

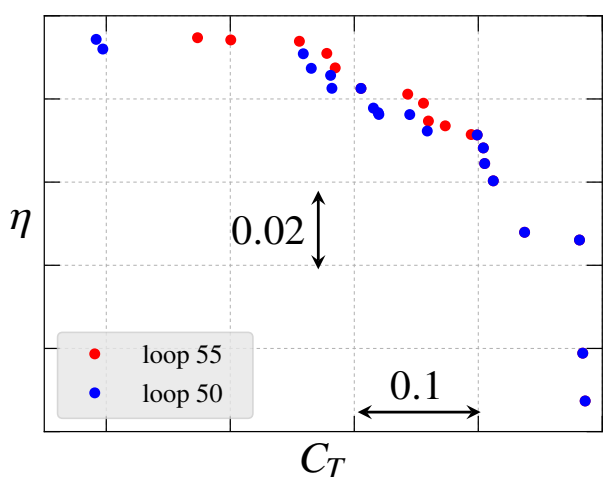

b) CROR with CST profiles.

Fig. 15 Real found pareto-fronts at different stages of the optimization.

$$
n_{2 n d}=\frac{(N+1)(N+2)}{2}
$$

which gives 1035 evaluations required for the 44 design variables in the NACA 16 parametrization, and 5995 for the 108 variables for the CST one. This clearly shows the much greater complexity of the CST problem, and reinforces the argument that the CST optimization had not reached its full potential at the time it was stopped.

\section{Conclusions}

Two independent aerodynamic CROR optimizations with different blade profiles have been performed using the automated platform for optimization HAMON, where multi-objective differential evolution coupled with radial basis functions and CFD evaluations is used. The optimization method applied has shown the capability of successfully improving the designs from the latin hypercube sampling, for both the NACA 16 and CST optimizations, by pushing towards designs with higher efficiency and thrust coefficient. The imposed limitation of using the same amount of computational resources has left the CST optimization non-converged and with unexploited improving potential, which the authors believe is the main reason why it has been outperformed by the NACA 16 optimization in this specific case.

\section{Acknowledgements}

The authors of this work would like to acknowledge the Swedish National Infrastructure for Computing (SNIC) for the computational resources provided at the High Performance Computing Center North (HPC2N) in Umeå, Sweden and the Chalmers Centre for Computational Science and Engineering $\left(C^{3} S E\right)$ in Gothenburg, Sweden.

\section{References}

[1] Parzych, D., Shenkman, A., and Cohen, S., "Large-Scale Advanced Propfan (LAP) Performance, Acoustic and Weight Wstimation," 1985.

[2] Stuermer, A., and Yin, J., "Low-Speed Aerodynamics and Aeroacoustics of CROR Propulsion Systems," 15th AIAA/CEAS Aeroacoustics Conference (30th AIAA Aeroacoustics Conference), 2009, p. 3134.

[3] Capitao Patrao, A., Avellán, R., Lundbladh, A., and Grönstedt, T., "Wake and Loss Analysis for a Double Bladed Swept Propeller," ASME Turbo Expo 2016: Turbomachinery Technical Conference and Exposition, American Society of Mechanical Engineers, 2016.

[4] Capitao Patrao, A., Montero Villar, G., Tomita, J. T., Bringhenti, C., Avellan, R., Lundbladh, A., and Grönstedt, T., "An Optimization Platform for High Speed Propellers," 2016. 
[5] Schnell, R., Yin, J., Voss, C., and Nicke, E., "Assessment and Optimization of the Aerodynamic and Acoustic Characteristics of a Counter Rotating Open Rotor,” Journal of Turbomachinery, Vol. 134, No. 6, 2012.

[6] Chen, N., Zhang, H., Xu, Y., and Huang, W., "Blade parameterization and aerodynamic design optimization for a 3D transonic compressor rotor," Journal of Thermal Science, Vol. 16, No. 2, 2007, pp. 105-114.

[7] Kulfan, B., and Bussoletti, J., "“" Fundamental" Parameteric Geometry Representations for Aircraft Component Shapes,” 11th AIAA/ISSMO multidisciplinary analysis and optimization conference, 2006.

[8] “GitHub repository for HAMON,” 2018. https://github.com/gmonterovillar/HAMON

[9] Montero Villar, G., Lindblad, D., and Andersson, N., "Multi-Objective Optimization of an Counter Rotating Open Rotor using Evolutionary Algorithms," 2018 Multidisciplinary Analysis and Optimization Conference, 2018.

[10] Ellbrant, L., Eriksson, L.-E., and Mårtensson, H., "CFD optimization of a transonic compressor using multiobjective GA and metamodels," Proceedings of the 28th International Congress of the Aeronautical Sciences, 2012, pp. 23-28.

[11] Pierret, S., "Multi-objective and multi-disciplinary optimization of three-dimensional turbomachinery blades," Sixth World Congress of Structural and Multidisciplinary Optimization, Rio de Janeiro, Brazil, May, Citeseer, 2005.

[12] Wang, X., Hirsch, C., Kang, S., and Lacor, C., "Multi-objective optimization of turbomachinery using improved NSGA-II and approximation model," Computer Methods in Applied Mechanics and Engineering, Vol. 200, No. 9-12, 2011, pp. 883-895.

[13] Lindsey, W., Stevenson, D., and Daley, B. N., Aerodynamic characteristics of 24 NACA 16-series airfoils at mach numbers between 0.3 and 0.8, National Advisory Committee for Aeronautics, 1948.

[14] Kulfan, B. M., "Universal parametric geometry representation method,” Journal of Aircraft, Vol. 45, No. 1, 2008 , pp. $142-158$.

[15] Spalart, P. R., and Allmaras, S. R., "A One Equation Turbulence Model for Aerodynamic Flows," 30th AIAA Aerospace Sciences Meeting and Exhibit, AIAA Paper 92-0439, 1992.

[16] Rai, M. M., "Single-and Multiple-Objective Optimization with Differential Evolution and Neural Networks," VKI lecture series: introduction to optimization and multidisciplinary design, Vol. 58, 2006.

[17] Deb, K., Pratap, A., Agarwal, S., and Meyarivan, T., "A Fast and Elitist Multiobjective Genetic Algorithm: NSGA-II," Evolutionary Computation, IEEE Transactions on, Vol. 6, No. 2, 2002, pp. 182-197.

[18] Zitzler, E., Deb, K., and Thiele, L., "Comparison of Multiobjective Evolutionary Algorithms: Empirical Results,” Evolutionary Computation, Vol. 8, No. 2, 2000, pp. 173-195. 